\title{
Reconstruction of Chronic Complicated Scalp and Dural Defects Using Acellular Human Dermis and Latissimus Dorsi Myocutaneous Free flap
}

Jun Hee Lee ${ }^{1}$,

Seok Keun $\mathrm{Choi}^{2}$,

Sang Yoon Kang ${ }^{1}$

Departments of ${ }^{1}$ Plastic and Reconstructive

Surgery and ${ }^{2}$ Neurosurgery, Kyung Hee

University School of Medicine, Seoul, Korea

No potential conflict of interest relevant to this article was reported.

\begin{abstract}
We present reconstruction of a complicated scalp-dura defect using acellular human dermis and latissimus dorsi myocutaneous free flap. A 62-year-old female had previously undergone decompressive craniectomy for intracranial hemorrhage. The cranial bone flap was cryopreserved and restored to the original location subsequently, but necessitated removal for a methicillin-resistant Staphylococcal infection. However, the infectious nidus remained in a dermal substitute that was left over the cerebrum. Upon re-exploration, this material was removed, and frank pus was observed in the deep space just over the arachnoid layer. This was carefully irrigated, and the dural defect was closed with acellular dermal matrix in a watertight manner. The remaining scalp defect was covered using a free latissimus dorsi flap with anastomosis between the thoracodorsal and deep temporal arteries. The wound healed well without complications, and the scalp remained intact without any evidence of cerebrospinal fluid leak or continued infection.
\end{abstract}

Keywords: Dura mater / Acellular dermis / Free tissue flaps
INTRODUCTION

A chronic complicated scalp and large dural defect is a complication that can follow neurosurgical procedures and poses a therapeutic challenge for clinicians. We present a case of reconstruction of a chronic complicated scalp and dural defect with a subdural abscess, using acellular human dermis and a latissimus dorsi myocutaneous free flap.

\section{Correspondence: Sang Yoon Kang}

Department of Plastic and Reconstructive Surgery, Kyung Hee University Hospital, Kyung Hee University School of Medicine, 23 Kyungheedae-ro, Dongdaemun-gu, Seoul 02447, Korea

E-mail: nuelk@nate.com

*This article was presented as a poster at the 69th Congress of the Korean Society of Plastic and Reconstructive Surgeons on November 11-13, 2011 in Seoul, Korea.

Received March 31, 2015 / Revised May 29, 2015 / Accepted August 4, 2015

\section{CASE REPORT}

A 62-year-old female presented with a cranial defect for reconstruction. Previously, the patient had undergone decompressive craniectomy for intracranial hemorrhage. The dura was closed with a synthetic polyurethane dural substitute (Neuro-Patch, B. Braun Melsungen AG, Melsungen, Germany), and after two months, the cranial defect was reconstructed using cryopreserved autologous cranial bone.

At three weeks after the cranioplasty, a yellowish exudative fluid was found to be discharge from the scalp incision, which was cultured and found to be a methicillin-resistant Staphylococcus aureus infection (Fig. 1A). On computed tomography (CT) scan, a fluid-filled dead space under the bone graft suspected to be the infectious nidus (Fig. 2A). Conservative management, including the administration of antibiotics, massive irrigation, and negative 
pressure wound therapy, did not obliterate this dead space. Three months after the initial operation, the grafted bone was removed, and the chronic ulcer was reconstructed using a bipedicled pericranial flap with the right superficial temporal artery as the pedicle, without removing the dural substitute.

One week after the pericranial flap coverage, the incision site began to discharge exudative fluid, and the patient was taken back to the operating room. On exploration, the infection tracked all the way to the dural substitute (Fig. 1B). Upon removal of the dural substitute, we found the space between the dural substitute and arachnoid membrane filled with exudative fluid, and the arachnoid was covered with a fibrotic, non-viable membrane. Debridement of this tissue revealed an $11 \mathrm{~cm} \times 9.5 \mathrm{~cm}$ dural defect. Two sheets of acellular human dermis $(4 \mathrm{~cm} \times 16 \mathrm{~cm}$ and $4 \mathrm{~cm} \times 12 \mathrm{~cm}$;
AlloDerm, LifeCell Corp., Branchburg, NJ, USA) were trimmed and fitted to close the dural layer in a watertight manner (Fig. 1C).

The dead space superficial dural layer was closed with an $11 \mathrm{~cm}$ $\times 5 \mathrm{~cm}$ latissimus dorsi myocutaneous free flap, from the right thorax. After primary closure of the donor site, the flap was inset, and the thoracodorsal artery was anastomosed to the deep temporal artery.

By one month following reconstruction, the wound had healed completely without further complications. On postoperative CT scan at 6-month follow up, subdural and epidural space was separated by the graft without dead space and fluid collection (Fig. 2B). The scalp defect was without any signs of further infection at 11month follow up (Fig. 1D).
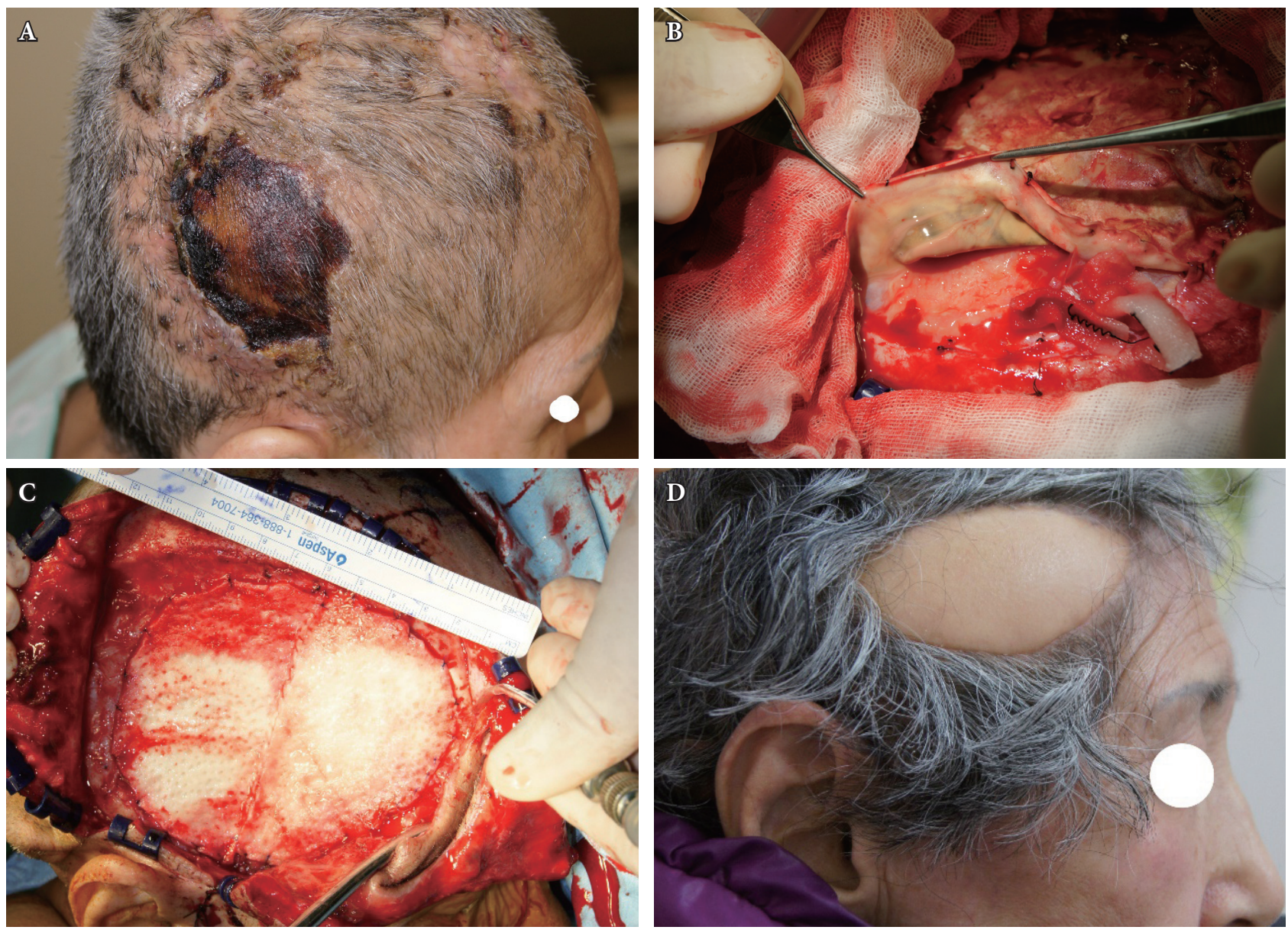

Fig. 1. Clinical photographs. (A) A $5 \times 4 \mathrm{~cm}$ eschar at the right temporoparietal scalp. (B) Exposed underlying prosthetic dura (Neuro-Patch). (C) Acellular human dermis used to close the dural defect over the right temporoparietal region. (D) Postoperative view of the scalp without further signs of infection or cerebrospinal fluid leak at 11 months after reconstruction. 

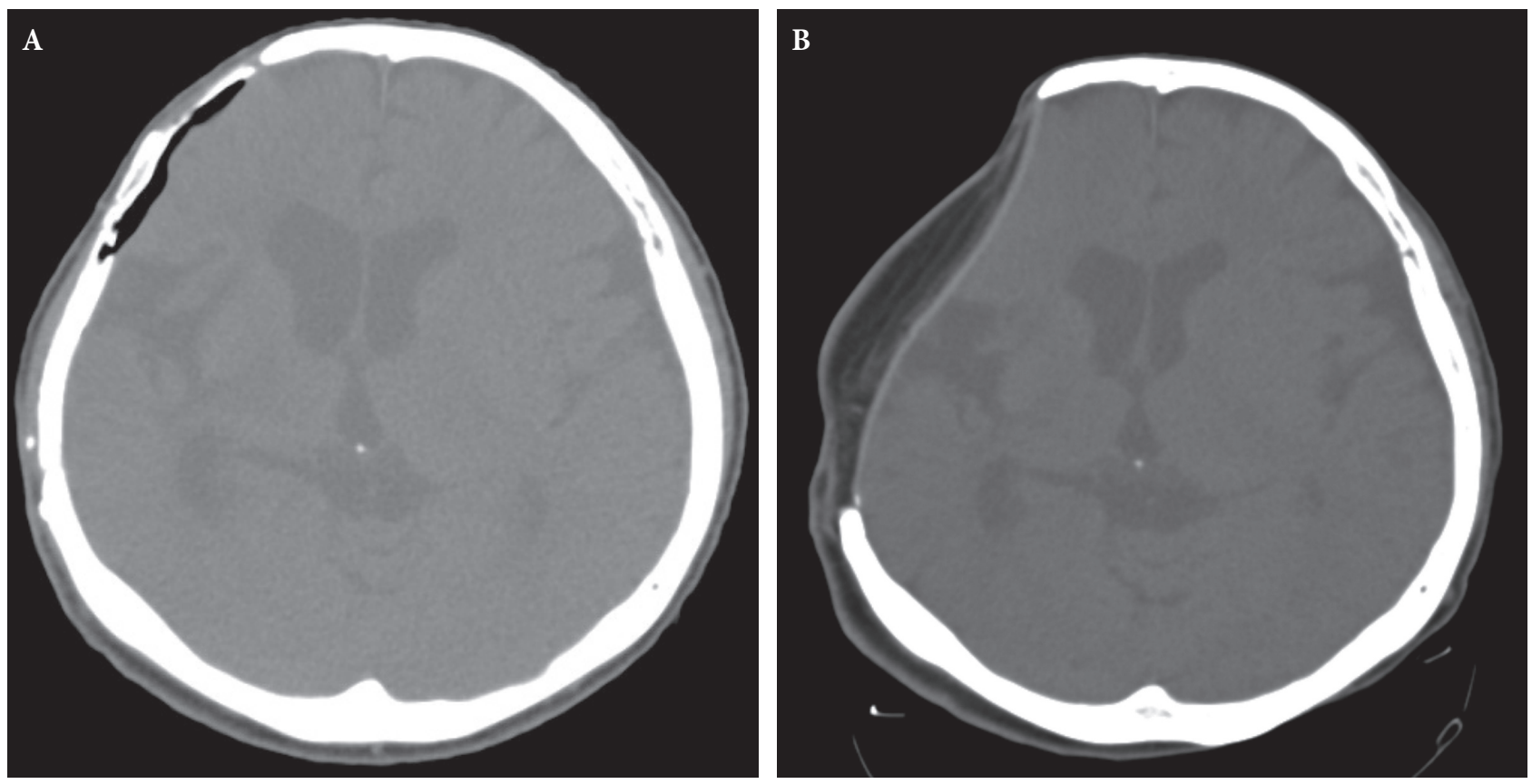

Fig. 2. Computed tomography (CT) images. (A) Preoperative CT scan demonstrating the abcess cavity under autologous bone graft. (B) Postoperative scan at 6 months showing separation of subdural and epidural space by the graft. No dead space and fluid collection is observed between flap and brain parenchyma.

\section{DISCUSSION}

Intractable chronic scalp ulcers can occur after cranioplasty. These lesions can result from severe scarring, poor blood circulation, and/or focal osteomyelitis. The treatment goal is to remove all infectious material and to replace the non-viable tissue $[1,2]$. If the infection involves a significant amount of dura mater, a replacement graft is required to maintain a watertight separation between the brain parenchyma and the scalp. The choice of graft material depends on the possibility of infection and availability of autologenous graft. Autografts of the pericranium or fascia remain the most widely accepted substitutes but are not always available or practical [2]. Xenografts, such as bovine pericardium, peritoneum, dermis, and flexor tendons, have also been used as dural substitutes [3,4], but can induce inflammatory reactions with rejection [2].

For our patient, we used acellular human dermis to maintain a seal subdural space. The graft is strong enough to withstand the suture tension required for watertight seal and does not cause cerebrospinal fluid leakage through the suture tracts. The graft is also soft, pliable, and elastic. It conforms to the cortical surface, providing an immediate watertight barrier for the brain. The graft is also readily available, easily prepared for use, and does not induce adhesion or rejection [2].

Acellular human dermis has two characteristic ultrastructural advantages for dural graft. First, all major histocompatibility class II antigens have been eliminated, which makes for an immunologically inert graft. Second, the extracellular structure allows for neovascularity, repopulation of cells, and ultimately the formation of neo-dura [2].

Free-flap reconstruction of extensive defects has been established to provide a sufficient quantity of tissue to fill dead spaces and restore the three-dimensional appearance of the head [5]. When reconstruction of extensive skull base defect is required, the latissimus dorsi free flap is a reliable solution, which can accommodate even the largest of defects. The thickness of the flap can be adjusted by removal of the deep fascia and subcutaneous fat tissue and provides a wide range of tissue composition (e.g. pure muscle flap, musculocutaneous flap with an independent and/or associated bone flap). In addition, the flap can be harvested with a long vascular pedicle, which can be useful in patients with 
damaged superficial temporal artery from a previous history of transcranial operation and/or injury. The majority of patients with latissimus dorsi flap donor site do not complain of shoulder pain, and there is no difference in the range of movement between the operated and the non-operated sides [5].

This case report describes a successful treatment of a complicated scalp and calvarial defect with a subdural abscess after cranioplasty. The dura mater was reconstructed using acellular human dermis graft, and the latissimus dorsi myocutaneous free flap was used to cover the remaining scalp defect. Acellular human dermis can be used to cover extensive dural defects, facilitate vascular ingrowth, decrease the propensity to incite inflammation and can be used at a previous site of infection [6]. Our case serve to illustrate that even the most complicated of infected cranial defects can be reconstructed using the combination of acellular human dermis graft and latissimus dorsi myocutaneous free flap.

\section{REFERENCES}

1. Boeckx WD, van der Hulst RR, Nanhekhan LV, De Lorenzi F. The role of free flaps in the treatment of persistent scalp osteomyelitis. Neurosurgery 59(1 Suppl 1):ONS64-7.

2. Warren WL, Medary MB, Dureza CD, Bellotte JB, Flannagan PP, Oh MY, et al. Dural repair using acellular human dermis: experience with 200 cases: technique assessment. Neurosurgery 2000;46:1391-6.

3. Anson JA, Marchland EP. Bovine pericardium for dural grafts: Clinical results in 35 patients. Neurosurgery 1996;39:764-8.

4. Xu BZ, Pan HX, Li KM, Chen XJ, Tian YD, Li YL, et al. Study and application of a porcine biomembrane for the repair of dural defects. J Neurosurg 1988;69:707-11.

5. Girod A, Boissonnet H, Jouffroy T, Rodriguez J. Latissimus dorsi free flap reconstruction of anterior skull base defects. J Craniomaxillofac Surg 2012;40:177-9.

6. Shridharani SM, Tufaro AP. A systematic review of acelluar dermal matrices in head and neck reconstruction. Plast Reconstr Surg 2012; 130(5 Suppl 2):35S-43S. 